Original Paper http://ajol.info/index.php/ijbcs http://indexmedicus.afro.who.int

\title{
Analyse de la diversité génétique des isolats de Magnaporthe oryzae du Burkina Faso et du Togo par les marqueurs microsatellites (SSRs)
}

\author{
Abalo Itolou KASSANKOGNO ${ }^{1,2^{*}}$, Ibrahima OUEDRAOGO ${ }^{2}$, Henri ADREIT ${ }^{3}$, Joël \\ MILAZZO $^{3}$, Leonard S OUEDRAOGO ${ }^{2}$, Philippe SANKARA ${ }^{1}$ et Didier THARREAU ${ }^{3}$ \\ ${ }^{1}$ Université Ouaga I Pr Joseph KI-ZERBO, Laboratoire Biosciences, 03 BP 7021 Ouagadougou 03, \\ Burkina Faso. \\ ${ }^{2}$ Institut de l'Environnement et Recherches Agricoles, BP 910 Bobo-Dioulasso, Burkina Faso. \\ ${ }^{3}$ Centre de Coopération Internationale en Recherche Agronomique pour le Développement, UMR BGPI TA A \\ 54/K Campus International de Baillarguet 34398 Montpellier Cedex 5 France. \\ *Auteur correspondant, E-mail : kassabalo@yahoo.fr
}

\section{RESUME}

La pyriculariose du riz est la principale maladie fongique la plus destructive de cette culture au Burkina Faso et au Togo. Afin d'élaborer une méthode de gestion durable de cette maladie, la connaissance de la diversité de l'agent pathogène s'avère nécessaire. L'objectif est d'acquérir des données sur la structure génétique des isolats de $M$. oryzae collecté entre 2013 et 2015 au Burkina Faso et au Togo. Treize (13) marqueurs microsatellites ont été utilisés pour caractériser 220 isolats selon la méthode décrite par Adreit et al. (2007). Les résultats révèlent un niveau de polymorphisme très élevé entre les isolats. Les valeurs moyennes de la diversité $(\mathrm{He})$ et des allèles par marqueur sont respectivement 0.55 et 9.36. Sur la base de la taille des séquences génotypes, 86 génotypes multilocus (MLGs) ont été identifiés. Ces résultats sont la conséquence probable de la diversité variétale du riz et de l'action environnementale sur l'expression des gènes chez le pathogène. Des MLGs sont partagés entre les deux pays et les pays voisins. Ce qui est probablement la conséquence directe des échanges de matériel végétal infecté entre ces pays. Ainsi, ces données couplées à la pathogénie de chaque génotype multilocus permettront l'amélioration des résistances de riz à la pyriculariose et les diverses relations identifiées nous inspirent des réflexions sur les stratégies de lutte contre M. oryzae excluant toute spécificité d'action dans la gestion de l'agent pathogène à l'intérieur d'un même pays.

(C) 2016 International Formulae Group. All rights reserved.

Mots clés : Caractérisation, Magnaporthe oryzae, diversité, microsatellites (SSRs).

\section{Analysis of the genetic diversity of Magnaporthe oryzae isolates from Burkina Faso and Togo by microsatellite markers (SSRs)}

\begin{abstract}
The blast is the most serious fungal disease, harmful to the cultivation of rice in Burkina Faso and Togo. In order to elaborate a methods of lasting management of disease, the knowledge a diversity of pathogen will be necessary. This study aims to collect data on the genetic structure within the isolates of M. oryzae collected between 2013 and 2015 in Burkina Faso and Togo. Thirteen (13) microsatellite markers were used to
\end{abstract}


characterize 220 isolates. The results showed a very high level of polymorphism between the isolates. The average values of gene diversity and allele frequency were 0.55 and 9.36 , respectively. Based on the size of the sequences genotypes, 86 genotypes multilocus (MLGs) were identified. These results are the probable consequence of the varietal diversity of rice and the environmental action on the gene expression. The common MLGs were identified between the two countries and the neighbour countries. What is probably the direct consequence of the exchanges of plant material infected by M. oryzae between these countries? Thus, these data coupled to pathogenesity of each genotype multilocus will improve rice resistance to blast and the various relationships we identified inspire reflections on control strategies of $M$. oryzae excluding any specificity action in the management of the pathogen within the same country.

(C) 2016 International Formulae Group. All rights reserved.

Keywords: Characterization, Magnaporthe oryzae, diversity, SSRs.

\section{INTRODUCTION}

La faible durabilité des résistances génétiques est liée à l'adaptation des agents pathogènes avec l'apparition et la sélection de nouvelles souches virulentes. Chez les champignons phytopathogènes, grâce à certaines caractéristiques telles que la sexualité et la mitose les permettent de s'adapter très rapidement à des modifications de leur environnement (McDonald et Linde, 2002), ce qui conduit à la diminution, voire à la perte de l'efficacité des moyens de lutte. Chez Magnaporthe oryzae, agent pathogène de la pyriculariose du riz la forte variabilité au sein de la population de l'agent pathogène est la principale raison de la faillite des résistances des cultivars de riz.

En effet, l'utilisation des variétés résistantes intégrant de bonnes pratiques agricoles qui était le moyen le plus efficace et le plus économique pour combattre la maladie est affrontée aujourd'hui aux phénomènes de contournement de la résistance des variétés (Fukuta, 2014 ; Kassankogno et al., 2015b). C'est pourquoi la connaissance de la structure génétique de la population de l'agent pathogène est nécessaire dans l'élaboration d'une méthode de gestion efficace et durable contre la maladie. Aujourd'hui, les microsatellites, connus sous le nom de « séquences simples répétées (SSR) » sont les plus appréciés des marqueurs moléculaires utilisés pour la détection du polymorphisme moléculaire chez $M$. oryzae. Ces marqueurs ont été utilisés dans plusieurs études de diversité de population de M. oryzae en Corée (Liu et al., 2008; Choi et al., 2013), en Iran (Brondani et al., 2000 ; Motlagh et al., 2015), au monde (Sahel et al., 2012). Cette étude a pour objectif de déterminer la structure génétique au sein des isolats de $M$. oryzae dans un but de mieux choisir les souches représentatives de la diversité existante dans les zones d'études pour les activités d'amélioration variétale.

\section{MATERIEL ET METHODES}

\section{Matériel fongique et culture mycélienne}

Une collection de 220 isolats monospores de $M$. oryzae réalisée pendant les campagnes rizicole 2014 et 2015 a été utilisée dans cette étude. Ces isolats proviennent des échantillons de feuilles ou de panicules de plantes de riz infectées qui ont été collectés dans plusieurs zones agro-écologiques au Burkina Faso et au Togo. L'isolement monospore et la purification ont été réalisés selon le protocole décrit par Hayashi et al. (2008). Le renouvèlement des isolats a été fait sur milieu farine de riz (composé de : $15.0 \mathrm{~g}$ d'agar, $20.0 \mathrm{~g}$ de farine de riz, $2.0 \mathrm{~g}$ d'extrait de levure, 1litre d'eau distillé et $1 \mathrm{ml}$ de pénicilline $\mathrm{G})$. La culture sur milieu liquide (composition e: 1 litre d'eau, $10.0 \mathrm{~g}$ de glucose, $3.0 \mathrm{~g}$ de $\mathrm{KNO} 3,2.0 \mathrm{~g}$ de $\mathrm{KH} 2 \mathrm{PO} 4$ et $2.0 \mathrm{~g}$ d'extrait de levure et autoclavé) a été faite à $25^{\circ} \mathrm{C}$. 
Extraction d'ADN et génotypage des isolats de Magnaporthe oryzae

L'extraction ADN a été faite suivant la méthodologie décrite par Adreit et al. (2007). Pour le génotypage, treize marqueurs microsatellites ou SSRs (Simple Sequence Repeats) définis par Kaye et al. (2003) et Adreit et al. (2007), ont été utilisés pour évaluer le niveau de polymorphisme des isolats étudiés (Tableau 1).

Les microsatellites ont été amplifiés par PCR multiplex en utilisant le kit multiplex QIAGEN. Les produits PCR ont été séparés par électrophorèse capillaire sur le séquenceur ABI 3700 (Applied Biosystems) de la plateforme Genseq du Labex CEMEB à l'Université de Montpellier (France).

\section{Analyse des données}

Cinq logiciels sont utilisés :

GeneMapper V4.1, a permet de convertir les pics d'amplification de chaque allèle taille d'amplicon. Ensuite les génotypes multilocus MLGs sont déterminés à l'aide de la macro Excel développée par Jean Peccoud. Puis avec PowerMarker 3.25 et Darwin 6.0 (Sokal et Michener), la description de la diversité génétique de la structure génétique est faite (Nei et al., 1983). Une analyse discriminante en composante principal DAPC a été réalisée en utilisant le «package » DAPC (Jombart et al., 2010) dans R (v2.13.1 R Development Core Team, 2008).

\section{RESULTATS}

Diversité génétique et structure des isolats de $M$. oryzae du Burkina Faso et Togo

L'analyse a révélé un taux de polymorphisme élevé pour 11 marqueurs avec 103 allèles détectés pour un nombre moyen de 9.36 allèles par locus. La fréquence des allèles majeurs varie de 0,14 pour Pyrms409 et 0,83 pour Pyrms319 avec une moyenne de 0,59.

Le Polymorphic Information Content (PIC) a varié de 0,24 pour Pyrms319 à 0,93 pour Pyrms409. La diversité génétique $(\mathrm{He})$ de Nei (ou hétérozygotie attendue) estimée pour chaque locus a varié entre 0,28 pour Pyrms319 et 0,94 pour Pyrms409 avec une moyenne de 0,55 (Tableau 2). De façon générale, on note une grande diversité génétique au sein des isolats étudiés.
L'analyse de la structure génotypique avec la macro excel, révèle 86.0 génotypes multilocus (MLGs) au sein des isolats analysés. L'assignation des 86 génotypes multilocus (MLGs) par pays donne une répartition suivante : 67 MLGs au Burkina Faso sur 185 isolats analysés équivalent à 36,21\%,16 MLGs au Togo sur 24 isolats analysés correspondant à $66,66 \%$ et 8 MLGs parmi les 11 isolats témoins inclus dans l'analyse. Distribution de groupes génotypes sur le Burkina Faso et sur le Togo 74.0.

\section{Distribution de groupes génotypes sur le Burkina Faso et sur le Togo}

Les résultats de la distribution des MLGs à travers le Burkina Faso (Figure 1) et le Togo sont les suivants: Au Burkina Faso les 67 groupes génotypes multilocus identifiés à travers le pays sont réparti comme suit : Dans les Hauts-Bassins, les MLGs suivant sont rencontré : $(1 *-8-13-19-20 \quad-21-22 *-23-$ $24-25-26-27-28 *-29-30-31-32-33-34-35-36-$ 37-38-39-41-42-43-44-45-46-47-48-49-50-5273-74-75-77-79-80). Dans les Cascades MLGs (1-8*-9-10-11-12-13-14-15-15-17-1828-35-51-52-53-54-55-56-57-58-59). Dans le Boucle du Mouhoun, on rencontre les MLGs : (1*-2-36-37-40). Dans le Sud- Ouest, on rencontre les MLGs : (28-37-39-41-78). Dans le Centre- Est, nous avons les MLGs : 1-3-45-6-8-76. Dans l'Est, nous avons les MLGs : (4-7) et dans le Centre-Nord, nous avons le MLG : (2). L'analyse comparative des génotypes multilocus de chaque région, fait ressortir un nombre de MLGs partagés entre certaines régions. Cependant cela n'exclut pas également des spécificités de groupes génotypes. Nous constatons par exemple que le MLG 1 se retrouve dans la région des Cascades, Boucle du Mouhoun, Centre-Est et Hauts-Bassins et le Centre-Est. De même, le MLG 8 est rencontre dans les régions du Centre-Est, Cascades, Hauts -bassins. Cependant on rencontre les MLGs 21, 22 uniquement dans la région des Hauts-bassins. $\mathrm{Au}$ Togo, sur les 16MLGs identifiés, on note une répartition comme suit à travers les régions du pays : la région des Savanes 5 MLGs (36-37-61-62-63), la région de la Kara (60-63-64-69-72); la région de la Centrale (12-62-65-67-70-71); la région des Plateau 
(36-66) et la région Maritime (36-66). Ce pays possède des MLGs partagés avec le Burkina Faso à savoir les MLG12, MLG36, MLG37 qui sont répartis sur l'ensemble du territoire togolais. Les chiffres précédés de signe astérix (*) sont les MLGs les plus importants de la région.

\section{Diversité inter-isolats du Burkina Faso et du Togo}

L'analyse de l'arbre de distribution des groupes génotypes multilocus identifiés parmi les isolats étudiés du Burkina Faso et du Togo, montre une certaine similitude génotypique entre les isolats provenant des deux pays. Cela suppose que les allèles amplifiés par les marqueurs au niveau de chaque loci sont identiques et égales.

Trois groupes génétiques sont distingués à savoir: G1, G2 et G3 (Figure 2). Ces groupes comportent des sous-groupes spécifiques notés A à M. Chaque sous-groupe comporte soit des isolats spécifiques d'un pays ou des isolats provenant de pays différents mais ayant des similitudes génotypiques proches.

De façon générale, on note que les groupes G1, G2 et G3 ont une base génétique commune mais très différenciés au niveau des loci amplifiés par les microsatellites.

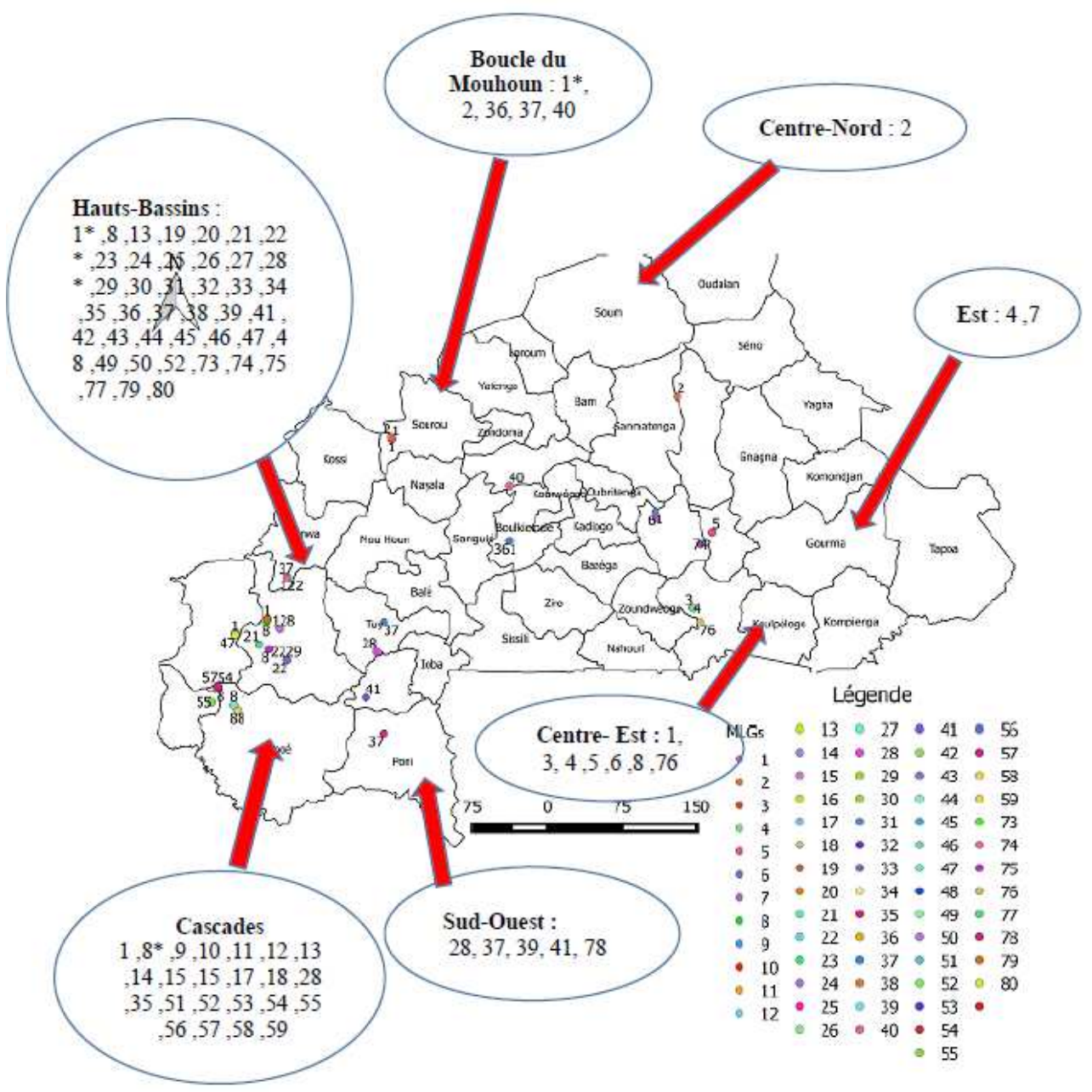

Figure 1 : Carte de distribution des génotypes multilocus dans les régions prospectées du Burkina Faso. 
Tableau 1: Caractéristiques des amorces microsatellites utilisés.

\begin{tabular}{|c|c|c|c|c|c|}
\hline Nom des amorces & Chromosome & Position & Motif répété & Séquences d'amorces & $\mathbf{T}_{\mathrm{m}}\left({ }^{\circ} \mathbf{C}\right)$ \\
\hline Pyrms43b-44 & 4 & 177(544920-544939) & $\mathrm{TA}_{12}$ & $\mathrm{~F}:$ :(NED)-GGGAATCTTGGCCATTAACC & 53 \\
\hline Pyrms409- 410 & 4 & 187 (520026-520045) & $\mathrm{TA}_{23}$ & $\begin{array}{l}\text { R : CTTGATTGGTGGTGGTGTTG } \\
\mathrm{F}: \text { (VIC)-TCCCAGTACTTGCCCATCTC } \\
\mathrm{R}: \text { CTCCGATTCATGGCACACAC }\end{array}$ & $\begin{array}{l}53 \\
53 \\
53\end{array}$ \\
\hline Pyrms99b-100 & 5 & $134(182811-182830)$ & $\mathrm{ACC}_{20}$ & $\begin{array}{l}\mathrm{F}: \text { (PET)-CTAATTGCGGCTTAGGGTCA } \\
\mathrm{R}: \text { ACCTAGGTAGGTATACATGTTGTT }\end{array}$ & $\begin{array}{l}53 \\
53\end{array}$ \\
\hline Pyrms63-64 & 1 & $9(243439-243601)$ & $\mathrm{CT}_{15}$ & $\begin{array}{l}\text { F: (NED)-TTGGGATCTTCGGTAAGACG } \\
\text { R: GCCGACAAGACACTGAATGA }\end{array}$ & 57 \\
\hline Pyrms83-84b & 2 & $18(211631-211742)$ & $\mathrm{TCA}_{13}$ & F: (PET)-GTCTGCCTCGACTCCTTCAC & 57 \\
\hline Pyrms319-320 & 2 & $18(52505-52795)$ & $\mathrm{CAA}_{6}$ & $\begin{array}{l}\text { R: GCAAAGTTGTTTGAGCAAGG } \\
\text { F: (NED)-TAAGACCACTGGCGGAATCT }\end{array}$ & 57 \\
\hline Pyrms77b-78 & 3 & $24(510594-510813)$ & $\mathrm{CA}_{24}$ & $\begin{array}{l}\text { R: GGCTTTGTCTGGTTGTACGG } \\
\text { F: (PET)-AGGCTCTCTGCCTACGAAGT } \\
\text { R: GCTTTCGGCAAGCCTAATC }\end{array}$ & 57 \\
\hline Pyrms607-608 & 3 & $28(1095575-1095864)$ & $\mathrm{GCA}_{13}$ & $\begin{array}{l}\text { F: (VIC)-CCCAAGCTCCATAATACGCTAC } \\
\text { R: TCCGAGACTCTTTGGATAGCAC }\end{array}$ & 57 \\
\hline Pyrms37-38 & 4 & $15(1042756-1042961)$ & $\mathrm{CA}_{6}+\mathrm{CT}_{12}$ & $\begin{array}{l}\text { F: (NED)-ACCCTACCCCCACTCATTTC } \\
\text { R: AGGATCAGCCAATGCCAAGT }\end{array}$ & 57 \\
\hline Pyrms47-48 & 4 & 12 (707029-707203) & $\mathrm{TA}_{15}$ & $\begin{array}{l}\text { F: (FAM)-TCACATTTGCTTGCTGGAGT } \\
\text { R: AGACAGGGTTGACGGCTGAA }\end{array}$ & 57 \\
\hline Pyrms233-234 & 5 & $10(38361-38617)$ & $\mathrm{CAG}_{10}$ & $\begin{array}{l}\text { F: (FAM)-TGAGATGGACCGCATGATTA } \\
\text { R: TTGATGGCAGAGACATGAGC }\end{array}$ & 57 \\
\hline Pyrms427-428 & 5 & $13(331827-332045)$ & $\mathrm{AT}_{16}$ & $\begin{array}{l}\text { F: (VIC)-CTGTCACCACAACCAAGACG } \\
\text { R: TTGCCCTGATTTGTCAGTCA }\end{array}$ & 57 \\
\hline Pyrms657-658 & 6 & $21(3745780-3745947)$ & $\mathrm{CA}_{12}$ & $\begin{array}{l}\text { F: (VIC)-ATCAGTCGAACCCACAAAGC } \\
\text { R: ATGTGTGGACGAACCAGTCC }\end{array}$ & 57 \\
\hline
\end{tabular}

Tm déterminé selon la formule : $\mathrm{Tm}=2^{\circ} \mathrm{C} \mathrm{x}($ nombre de $[\mathrm{A}+\mathrm{T}])+4^{\circ} \mathrm{C} \mathrm{x}($ nombre de $[\mathrm{G}+\mathrm{C}])$. 
Tableau 2: Description de la diversité des isolats génotypés pour chaque loci.

\begin{tabular}{lllll}
\hline & Fréquence des & & & \\
Marqueurs & Allèles majeurs & A & He & PIC \\
\hline Pymrs47 & 0.38 & 14.00 & 0.71 & 0.66 \\
Pyrms427 & 0.25 & 10.00 & 0.83 & 0.81 \\
Pyrms657 & 0.67 & 5.00 & 0.49 & 0.43 \\
Pyrms77B & 0.68 & 12.00 & 0.52 & 0.50 \\
Pyrms63 & 0.74 & 8.00 & 0.41 & 0.37 \\
Pyrms83B & 0.66 & 8.00 & 0.51 & 0.47 \\
Pyrms607 & 0.74 & 5.00 & 0.42 & 0.39 \\
Pyrms37 & 0.69 & 6.00 & 0.45 & 0.37 \\
Pyrms233 & 0.67 & 7.00 & 0.49 & 0.43 \\
Pyrms319 & $\mathbf{0 . 8 3}$ & $\mathbf{2 . 0 0}$ & $\mathbf{0 . 2 8}$ & $\mathbf{0 . 2 4}$ \\
Pyrms409 & $\mathbf{0 . 1 4}$ & $\mathbf{2 6 . 0 0}$ & $\mathbf{0 . 9 4}$ & $\mathbf{0 . 9 3}$ \\
Total & & 103 & & \\
Mean & $\mathbf{0 . 5 9}$ & $\mathbf{9 . 3 6}$ & $\mathbf{0 . 5 5}$ & $\mathbf{0 . 5 1}$ \\
\hline
\end{tabular}

F allèles majeurs : Fréquence des allèles majeurs; $\mathbf{A}$ : nombre d'allèles, He : hétérozygotie attendue calculée sans biais ou diversité génétique ; PIC : Polymorphism Information Content.

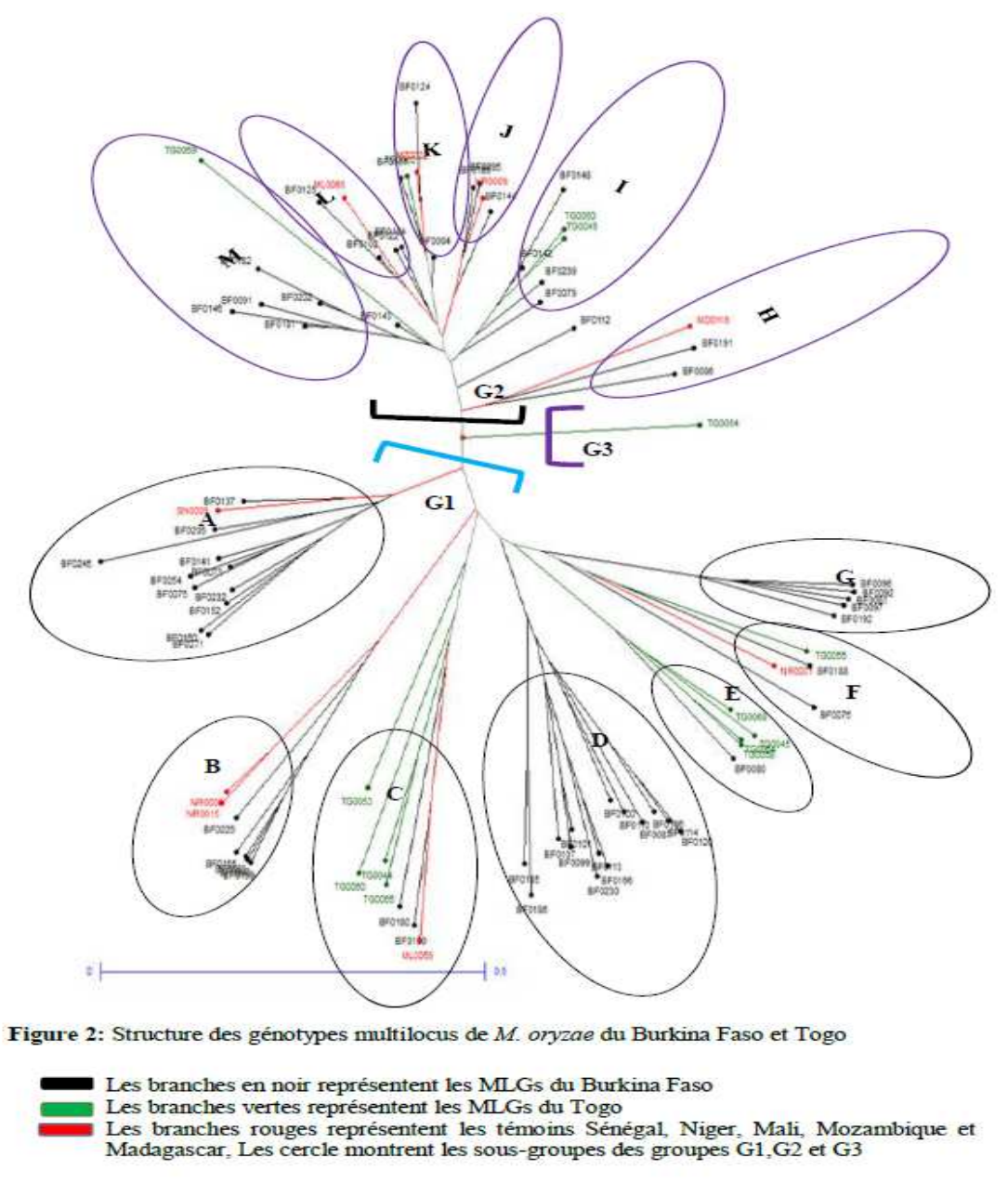




\section{DISCUSSION}

Nous avons étudié le polymorphisme de 220 isolats de Magnaporthe oryzae du Togo et du Burkina Faso avec des marqueurs microsatellites (SSRs). Les résultats de l'étude montrent un polymorphisme élevé entre les isolats testés. Les SSRs utilisés ont permis de détecter 103 allèles avec un nombre moyen de 9 allèles par marqueur. Le nombre d'allèles par marqueur a varié de 2 à 26 respectivement avec Pyrms 319 et Pyrms 409. La fréquence des allèles majeurs varie de 0,14 à 0,83 respectivement avec Pyrms 409 et Pyrms 319 avec une moyenne de 0,59. Nos résultats différents en termes de richesse allélique de ceux de Liu et al. (2008) et Choi et al. (2013) qui ont étudié respectivement la diversité génétique de 46 isolats et 109 isolats de $M$. grisea du Corée avec respectivement 13 marqueurs et 11 marqueurs SSRs. Ces auteurs ont trouvé respectivement dans leur étude entre 2-10 allèles et 2-17 allèles par locus.

L'analyse des données de la diversité génétique entre les isolats a révélé un $\mathrm{He}$ variant entre 0,24 et 0,94 , ce qui montre un très grand polymorphisme généré par les marqueurs utilisés. La grande diversité observée dans notre étude s'expliquerait par l'effet de l'environnement dans les zones d'étude qui aurait favorisé expression d'autres gènes et la diversité variétale qui aurait favorisé la diversité au sein de la population du pathogène.

L'analyse du polymorphisme en se basant sur le «Polymorphisme Information Content » (PIC) considéré comme le premier index important dans la détermination de l'efficacité des amorces a donné un PIC compris entre 0,24 et 0,93 respectivement enregistré par les marqueurs pyrms 319 et pyrms 409 avec une moyenne de 0,51. Nos résultats diffèrent peu de ceux de Motlagh et al. (2015), de Brondani et al. (2000) qui ont trouvé des PICs compris respectivement entre 0,661-0,862 et 0,44-0,54 lors de l'analyse des isolats de M. oryzae Iranien. Cette variation de PIC observée dans notre étude est significativement associée au nombre d'allèles observés par marqueur. L'analyse de la diversité génétique mesurée par l'indice $\mathrm{He}$ (ou hétérozygotie attendue) a été estimée pour chaque locus. La plus faible valeur de diversité génétique $(0,28)$ a été enregistrée par le locus pyrms 319 et la plus forte valeur $(0,94)$ par le locus pyrms 409 avec une moyenne de 0,55 . Le nombre d'allèles $(A=26)$ et la diversité génétique $(\mathrm{He}=0,94)$ trouvés dans notre étude sont supérieurs à ceux trouvés par d'autres auteurs. Nos résultats ont révélé une plus grande diversité par rapport à ceux trouvés par Choi et al. (2013) lorsqu'ils ont étudié la diversité des isolats Coréens de M. oryzae avec 11 amorces microsatellites. En effet, ceux-ci ont trouvé A moyen de 6.5 et $\mathrm{He}$ de 0,412 contre 26 et 0,94 respectivement dans notre étude. Cette différence des résultats pourrait s'expliquer par le fait qu'en Corée, il y'a une faible diversité de riz cultivé par rapport au Burkina Faso et au Togo. En effet, le système rizicole en Afrique de l'Ouest et plus particulièrement au Burkina Faso et au Togo est caractérisé par la culture plurivariétale. Cette pratique qui permet d'avoir un minimum de rendement quelle que soit l'influence des facteurs biotiques et abiotiques sélectionne aussi plus de diversité chez les pathogènes. Notre isolement étant réalisé sur plusieurs variétés de riz ce qui expliquerait la diversité constatée dans notre étude.

L'analyse moléculaire a permis de comparer les isolats en fonction de leur similitude sur la base de la taille des séquences génotypes. À l'aide de la macro Excel développée par Jean Peccoud, les souches ont été regroupées en génotypes multilocus (MLGs).

Ainsi, pour 13 marqueurs polymorphes utilisés, nous avons identifié 86 génotypes multilocus.

Nos résultats diffèrent de ceux de Sahel et al. (2012) qui avaient trouvé un faible nombre de génotypes multilocus lors de son étude: Thaïlande (20 MLGs), Chine (5-76 MLGs), Colombie (9 MLGs), France (12 MLGs), USA (20 MLGs) et Madagascar (18 MLGs). Également ces résultats présentent une plus grande diversité génotypique par rapport à ceux de Consolo et al. (2008) et de Ying et al (2004), qui ont étudié 
respectivement la diversité génétique de 161 isolats de $M$. oryzae de l'Argentine et 105 isolats de Chine avec sept (7) SSRs. Les premières ont trouvé cinq (05) groupes génotypes avec une similitude élevée (plus de $70 \%$ ) alors que les seconds ont rapporté six (6) groupes génotypes. Sur les 86 génotypes multilocus identifiés parmi les isolats, le Burkina Faso a enregistré 67 MLGs et le Togo 16 MLGs. Ces nombres important de MLGs serait lié soit à la diversité variétale du riz ayant servi pour l'isolement des isolats ou lié à la sélection naturelle due à l'écologie des sites. Nous avons noté un partage de génotypes multilocus au sein d'un même pays et entre les pays. Le cas des MLG1 et MLG8 entre certaines régions du Burkina Faso et le cas du MLG12 ; MLGs36 et MLG37 partagés entre le Burkina et le Togo. Nous pensons que ce résultat est la conséquence directe des échanges de matériel végétal entre les deux pays.

\section{Conclusion}

L'étude a apporté un nouvel éclairage sur la structure des populations de $M$. oryza au Burkina Faso et au Togo. Un niveau de polymorphisme élevé entre les isolats a été révélé.

Les diverses relations identifiées entre les groupes génotypes multilocus, les groupes génétiques mondiaux et les sites, nous inspirent des réflexions sur les stratégies de lutte contre $M$. oryzae et sur la dynamique des populations de l'agent pathogène dans le temps dans ces deux pays. Les similitudes génotypiques rencontrées entre certains isolats du Burkina Faso, du Togo et des autres pays pourrait être la conséquence directe des échanges de semences infectées entre ces pays. Ainsi dans un contexte des échanges mondiaux intenses, les connaissances sur la structure et la diversité chez $M$. oryzae devraient conduire à une vigilance accrue sur le risque d'introduction de nouvelles races du pathogène à travers les échanges de semences de riz. Dès lors des investigations devraient être menées dans l'avenir sur le rôle des semences dans la propagation de la maladie ainsi que la détermination des seuils de nuisibilité du pathogène sur les semences.

\section{CONFLIT D'INTERETS}

Les auteurs déclarent qu'il n'y a aucun conflit d'intérêts.

\section{CONTRIBUTION DES AUTEURS}

AIK est l'investigateur principal du projet de recherche et a rédigé le manuscrit. IO a été le superviseur direct des travaux de collecte, de réalisation d'isolats monospore et la rédaction du manuscrit. JM et HA ont aidé à faire l'extraction des ADNs, les PCRs multiplex et à la transformation des données avec GeneMapper après génotypage. LSO a été notre co-encadreur scientifique et a corrigé le manuscrit. PS a été l'encadreur scientifique au niveau de l'école doctorale Sciences et Technologies de l'Université Ouaga I Pr Joseph KI-Zerbo. DT a été le superviseur de l'ensemble des travaux moléculaires réalisé à BGPI-CIRAD Montpellier (France) et a aidé à l'analyse des données.

\section{REMERCIEMENTS}

Les auteurs remercient l'équipe du laboratoire de phytopathologie INERA-Bobo (Burkina Faso), l'équipe $5 \mathrm{du}$ laboratoire BGPI-CIRAD Montpellier (France), la plateforme Genseq du Labex CEMEB à l'Université de Montpellier (France), Le Bioinformaticien de BGPI Sébastien RAVEL pour leur soutien dans la réalisation de ce travail.

\section{REFERENCES}

Adreit H, Santoso, Andriantsimialona D, Utami DW, Notteghem JL, Lebrun MH, Tharreau D. 2007. Microsatellite markers for population studies of the rice blast fungus, Magnaporthe grisea. Molecular Ecology Notes, 7: 667-670.

Choi J, Park SY, Kim BR, Roh JH, Oh IS, Han SS, Lee YH. 2013. Comparative Analysis of Pathogenicity and Phylogenetic Relationship in Magnaporthe grisea Species Complex. PLoS ONE, 8(2): e57196.

Consolo VF, Cordo C.A, Salerno G.L. 2008. 
DNA fingerprint and pathotype diversity of Pyricularia oryzae populations from Argentina. Australas. Plant Pathol., 37: 357-364.

Hayashi N, Kobayashi N, Vera Cruz CM, Fukuta Y. 2009. Protocols for the sampling of diseased specimens and evaluation of blast disease of rice. Japan international research center for agricultural science. Working Report, 63: 7-28.

Jombart T, Devillard S, Balloux F. 2010. Discriminant analysis of principal components: a new method for the analysis of genetically structured populations. BMC Genetics, 11: 94.

Kassankogno AI, Saibou A, Ouedraogo I, Kpemoua KE, Nana A, Ouedraogo L, Sankara P. 2015b. Caractéristiques morphologiques et phénotypiques des isolats de Magnaporthe grisea du Burkina Faso et du Togo 17p Afrique Science, 11(4): 361 - 377.

Kaye C, Milazzo J, Rozenfeld S, Lebrun MH, Tharreau D. 2003. The development of simple sequence repeat markers for Magnaporthe grisea and their integration into an established genetic linkage map. Fungal Genetics and Biology, 40: 207214.

McDonald BA, Linde C. 2002. Pathogen population genetics, evolutionary potential, and durable resistance. Annu.
Rev. Phytopathol., 40: 349-379.

Nei M. 1973. Analysis of gene diversity in subdivided populations. Proc. Natl. Acad.

Saleh D, Xu P, Shen Y, Li C, Adreit H, Milazzo J, Ravigné V, Bazin E, Notteghem JL, Fournier E, Tharreau D. 2012. Sex at the origin: an Asian population of the rice blast fungus Magnaporthe oryzae reproduces sexually. Molecular Ecology, 21: 1330-1344.

Sere Y, Onasanya A, Afolabi A, Mignouna HD, Akator K. 2007. Genetique diversity of the blast fungus, Magnaporthe grisea (Hebert) Barr, in Burkina Faso. Africain Journal of Biotechnology, 6(22).

Ying S, Froun J, Yue-Qiu H, Kaya C, FangHua $X$, Notteghem JL, Er-Ming L, Tharreau D. 2004. The perfect stage and SSR analysis of Magnaporthe grisea in Yanxi blast nursery. Chinese J. Rice Sci., 18(3): 262-268.

Fukuta Y, Koga I, Ung T, Sathya K, Kawasaki-Tanaka A, Koide Y, Kobayashi N, Obara M, Yadana H, Hayashi N. 2014. Pathogenicity of Rice Blast (Pyricularia oryzae Cavara) isolates from Cambodia . JARQ $\quad$ 48(2): 155-166. http://www.jircas.affrc.go.jp 